\title{
PRÁCTICAS CORPORALES. TRADUCCIÓN DE SENTIDOS EN LA CIUDAD
}

\author{
Gabriel Armando Cachorro \\ Facultad de Humanidades y Ciencias de la Educacion de La Plata, Argentina.
}

\begin{abstract}
Resumen
Este trabajo está referido al relevamiento e inventario de prácticas corporales desplegadas por los/las jóvenes en la ciudad de la Plata. El levantamiento de estos materiales en el trabajo de campo es aprovechado para un análisis interpretativo de los sentidos sociales puestos en juego por los/las jóvenes con el dibujo de mapas y cartografías de los modos de participación de ellos. La propuesta del trabajo aspira a construir una estética de la mirada capaz de acompañar los procesos de construcción de la ciudadanía a través de ese precioso material llamado cuerpo.
\end{abstract}

Palabras claves: Prácticas corporales - Ciudad - Jóvenes - Cuerpo.

\section{Las prácticas y los deseos}

L as prácticas corporales de los sujetos constituyen instancias de materialización de deseos. Si los sujetos comparten un espacio en la ciudad es porque existe una necesidad de estar al lado de alguien, encontrarse y expresar "modos de estar juntos" (MAFFESOLI, 2004, p. 17). Cada vez que encontramos personas compartiendo un recinto social desplegando alguna actividad con un compromiso directo del cuerpo contamos con la posibilidad de analizar los elementos que se ponen en juego en las relaciones humanas y las excusas que argumentan una reunión entre participantes.

Las prácticas corporales están movidas por anhelos de construir tramas vinculares. Los espacios de participación ciudadana generados cobijan sueños y tratan de justificar la propia existencia. Vivir esas prácticas corporales en la ciudad con intensidad, sentir la vibración de la convivencia con otros diferentes constituye una cabal certificación de que estamos dentro de la historia y somos parte de algo. Las prácticas corporales están modeladas por fuerzas ingobernables (amores, odios, complicidades, simpatías, aversiones), con estructuras que se imponen a las "sensibilidades vulnerables” (LEVINAS, 1987, p. 201), en esa capacidad de afectar y ser afectado.

No estar fuera de la vida, ser integrante de grupos sociales de las prácticas corporales en la ciudad, asegura la consolidación de núcleos de referencia y pertenencia en "los procesos de identificación" (GALLISOT, 1987). La referencia como residencia espacial donde se produce la frecuenta y la pertenencia como vínculo humano con otros que otorga la felicidad de formar parte de un grupo. El deseo social, la reali- 
zación personal en un colectivo de personas encuentra en las prácticas corporales un sitio valioso para materializar proyectos de vida. La imposibilidad de estar solo y la invención de cosas para encontrar eventos sociales o aprovechar los existentes otorga sentido y amarra al sujeto a algo de que sostenerse.

Las prácticas corporales tienen el valor de hacer. Hacer que pase algo. Ese pasar como acontecimiento es muy importante porque no deja al sujeto en el aire. En las experiencias sociales memorables y las anécdotas originales, los episodios trágicos, la aventura romántica, se produce algo más que un cúmulo de experiencias de vida incrustadas en el cuerpo. La ostentación de tours sexuales, la participación exitosa en campeonatos de futbol infantil, el logro de un cinturón negro, el record de salto en largo, la experiencia de supervivencia en una isla, la escalada a una cumbre peligrosa, las epopeyas corporales heroicas pasan a ser episodios del cuerpo valiosos en el trayecto de vida, otorgan distinción, marcan una diferencia del resto. Siguiendo la sugerencia de Gagliano (2005, p. 208) de dar "agasajos y bienvenidas" podemos "ritualizar" esas prácticas para adosarle jerarquía y generar recuerdos capaces de grabar eventos en la memoria de los practicantes, cuidando las situaciones corporales y motrices, resaltándolas como importantes.

La construcción de estas historias de vida pasadas por la experimentación corporal ayuda a evitar la nada o el vacío como pobreza existencial de haber comprobado el desperdicio del tiempo individual. Si existe esta preocupación de que algo pase es porque es necesario llenar de vida a los terrenos vírgenes y no estar con la sensación de ser un sujeto con un mundo interno hueco o empobrecido. Esta carencia de cosas para contar implica soportar la expresión categórica "No existís" en el auditorio. La escritura de la historia pasa por darle cuerpo a las palabras y nombrar eventos con un relato de autor en primera persona.

\section{Interrogar las prácticas corporales de la ciudad}

Las prácticas corporales desplegadas en la ciudad sirven para consolidar relaciones y contactos sociales entre parientes, compañeros de trabajo, generando un encuadre de la relación diferente al ofrecido por el tiempo productivo. Las prácticas deportivas ofrecen agonismo, competencia, tensión y establecen una prolongación de las instancias de la vida ordinaria y vulgar que a veces desarrollamos en nuestros mundos de vida. Es un espacio que también posibilita enfermarse más, contracturarse, infartarse y tener diversos contratiempos físicos. También buscamos o al menos merodeamos la muerte y la destrucción. Es un espacio lúdico, es un juego social que todos queremos jugar. En el juego encontramos la posibilidad de reinventarnos en el disfrutar motorizando nuestras subjetividades. En esas prácticas corporales se exteriorizan solidaridades, servidumbres, miserias, honestidades, mecanismos de poder, enfermedades y en realidad eso es lo que se pone en juego. En la práctica corporal se pone la densidad de la subjetividad a través del cuerpo. Por lo tanto se juegan cosas distintas para cada participante.

La necesidad de estar con otros se renueva constantemente. Se exterioriza al pertenecer a cofradías, tener espacios de reconocimiento motriz, recibir las felicitaciones por logros deportivos o elogios por maniobras técnicas, exhibir ejecuciones indivi- 
duales habilidosas en el juego motor, progresar en logros de competencias motrices. Son espacios de felicidad corporal obtenidas en momentos esporádicos. Se halla a veces en las propuestas de arraigo populares. Cada quien es feliz como quiere y como puede. El levantador olímpico de pesas que se pliega sobre si mismo en su ostracismo corporal de la abstinencia, la dieta y la dosificación de sus fuerzas físicas; el disciplinado ciclista que toma como gran referente a Curuchet pedaleando al infinito, el obsesivo futbolista que remite todo a una pelota, el vocacional bailarín de ballet provisto de una sensibilidad a contrapelo de las mayorías machistas o el apasionado amante de todo bicho que camina. En esa diversidad de prácticas corporales las apuestas son distintas, pero convergen en la búsqueda de sentidos.

La alegría de un sábado se resume a veces en la posibilidad de ganar un partido de futbol amistoso de carácter informal. Encontrar explicaciones a la relación de los sujetos con sus cuerpos implica ponerse en una postura abierta a una trama cultural ajena, desprovista de prejuicios y reduccionismos simplistas, tal vez inscribiéndonos en la perspectiva de Armando Silva (2005) con sus "Polvos de ciudad". El lugar que se le da al cuerpo en la ciudad se liga a móviles autobiográficos. Esos móviles tienen que ver con las ambiciones de trascendencia individual, los planos de realización que cada ciudadano fantasea para no pasar desapercibido ante los demás. Lo peor que le puede pasar al sujeto es tener un pobre recorrido existencial. No ser reconocido, ser un fantasma que no despierta absolutamente nada, pasar sin ser nombrado, generar la pura intrascendencia. Ser nada, ser pura indiferencia e irrelevancia. No tener algo que dejar para los otros muestra la pobreza de la subjetividad o la irresponsabilidad de descuidarla como lo advierte Silvia Bleichman (2005, p. 85) en los procesos de "dessubjetivación" en nuestra sociedad y la alarma de proponer "modos posibles de recomposición".

\section{Modos de ser ciudadanos prácticos}

Recuperando a Duschatsky (2005, p. 224), podemos diferenciar la idea del cuerpo como instancia física a la intemperie o el cuerpo simbólico de una institución que sujeta a varios. No solo alcanza con el mero estar, es necesario superar esa instancia y pasar al estar siendo. Ese cambio significa saltar de la platea al escenario, involucrarse en una mayor proximidad que movilice la opinión, la palabra que se juegue y arriesgue haciendo mención a los cuerpos. La posibilidad de construir a través de un compromiso político con posibles niveles de conciencia ciudadana exige hechos corporales en vez de promesas. Los ciudadanos, para desenvolverse en los laberintos urbanos, conviven con miedos, peligros, acosos, violencias, seducciones, neurosis, histerias, fastidios. La ciudad y el cuerpo mantienen esa relación subjetiva. Es una ficción a veces pesimista y otras tantas optimistas de estar en ella. Parafraseando a Lacarrieu y Pallini (2007), podemos “imaginarnos Buenos Aires” desde otra perspectiva de la mirada.

La seducción del peligro, el coqueteo con la muerte, la provocación del límite reglamentario, son elementos desplegados en algunas prácticas corporales de la ciudad. La experimentación del vértigo, la aceleración de las pulsaciones, las descargas adrenérgicas de actividades corporales que arriesgan la integridad física tal vez tengan puntos de relación con el aura de la ciudad. La densidad de algunos barrios, el clima 
pesado de la noche turbia, las balaceras de zonas liberadas, la latencia de apariciones sorpresivas de violadores, delincuentes, secuestradores, elementos que empapan a los ciudadanos de niveles de pánico, miedo, terror, horror, perversión, morbosidad, entre otras propiedades sujetas a infinitas combinaciones, en la construcción siempre incompleta de la inalcanzable subjetividad.

Las prácticas corporales del pan kur exponen no solo el riesgo de caerse en el salto de un techo a otro, también incluyen el peligro concreto de ser derribado por un intolerante vecino asumiendo el carácter de francotirador, o ser denunciado a agentes policiales por el "buchón” de esas destrezas corporales aéreas. La reflexividad corporal está en esas formas violentas de poner el cuerpo en una ciudad peligrosa. Lanzarlo al vacío, provocar el susto, vivir la tentación de transgredir significa algo más. Según Paul Virilio (1997, p. 48), en ese tipo de actividades corporales "la perspectiva acelerada prevalece sobre la perspectiva pasiva de los perspectivistas". Gozar de la pelea, la tortura, la humillación al otro en el plano de la degradación física se expresa en la violencia urbana. La práctica corporal a veces busca destrucción, suplicio, tortura, maltrato, calvario. Deja entrever formas de relación de los sujetos entre sí. En esa línea interpretativa, los trabajos de José Luis Barrios (2008) sensibilizan una mirada interesante anclada en lo grotesco, el asco, el morbo, en la cultura que vivimos actualmente. Hacer sufrir, hacer agonizar, son vectores de la subjetividad ejecutados en la carne de los sujetos.

¿Dónde y cómo ponen el cuerpo y la subjetividad los sujetos practicantes? ¿En qué queman sus energías? ¿Cuáles son sus espacios de placer, qué actividades les entregan felicidad, realización existencial? ¿Por dónde circulan las angustias, frustraciones, proyectos de vida de los ciudadanos? ¿Qué les quita el sueño a los adeptos a la gimnasia aeróbica, los fervientes simpatizantes de algún equipo deportivo, como se engendran fantasías, sueños, satisfacciones y frustraciones? Nuevamente las prácticas corporales desplegadas en la ciudad se ofrecen como puertas de acceso a la subjetividad.

Nuestra tarea como investigadores radica en la difícil misión de decodificar, con todos los procedimientos heurísticos del descubrimiento, los códigos y lógicas de manifestación puestas en clave. En esa línea estamos trabajando para hacer un inventario de las propuestas corporales de la ciudad y para aplicar un enfoque interpretativo cualitativo sobre esos materiales recolectados para su posterior catalogación (proyecto de investigación "El campo de las prácticas corporales en la ciudad de La Plata”, 2008-2011).

\section{Prácticas corporales discontinuas}

Las prácticas corporales constituyen puestas en acto de esquemas de pensamiento, matrices culturales, "frames", marcos de referencia. No operan sin teorías. Están guiadas en la perspectiva de Bourdieu (1984) por habitus, es decir "estructuras estructuradas predispuestas a actuar como estructuras estructurantes". Este concepto, entonces, rebasa la noción de mero hacer instrumental o ejecución automática de actividades corporales alienadas. La relación de los sujetos con las prácticas corporales entendida con continuidad, en el sentido de incorporar una rutina con regularidad o sistematicidad de la actividad física, es una premisa difícil de lograr. 
La persistencia, la disciplina corporal, es un punto conflictivo, de acuerdo con los dueños de gimnasios, los maestros de artes marciales, o quienes enseñan clase de bailes afroantillanos. La relación del sujeto con su cuerpo ofrece esos desencuentros y encuentros. Abandonos temporarios, cambios de propuestas, pruebas de opciones corporales a la carta. Es un elemento que provoca la revisión de las propuestas ofrecidas a los practicantes. ¿Por qué dejan? ¿Dónde está la falla? No es tan fácil cumplir con aquel objetivo de la gimnasia de "mejorar la relación del sujeto con su cuerpo y su cultura". En realidad, en lo profundo, ese objetivo es demasiado ambicioso en su extensión.

Alguna vez hablaba con un especialista en la enseñanza de un arte marcial y me comentaba de estos abandonos y de lo poco que le duraban sus aspirantes, también destacaba el importante número de personas que había incursionado a lo largo del año en cortos lapsos de tiempo desechando con rapidez la práctica física. El tema por analizar en esos tipos de organizaciones sociales son los modos de relación de poder y saber que atraviesan las relaciones entre formadores y aprendices de una disciplina corporal. Esa situación es central para interpretar compatibilidades e incompatibilidades entre los actores involucrados. Los escenarios sociales de los seguidores del Yoga, el Kung Fu, el Aikido, ameritan lecturas complejas.

La asimetría de las relaciones en los procesos de formación se refuerza con el abuso de solicitudes de tareas fuera de las destrezas corporales y motrices. Que un aprendiz de kung fu, después de hacer su sesión diaria, tenga que quedarse a barrer el piso, podar una planta o cumplir con un castigo físico por desobedecer una consigna del maestro encuentra respuestas a la medida de los narcisismos y las subjetividades provocadas. Una mente débil, manejable, dócil, constituye una materia prima virgen para ser modelada a gusto del escultor.

¿Qué busca un joven en un arte marcial? Sospechamos que una experiencia distinta, la entrada a la excentricidad antropológica, la exploración de lo raro, la curiosidad por lo inédito. Estos grupos son sitios capaces de aprovechar subjetividades a la deriva para introducir en sus cabezas los disparates más inverosímiles. Charlas de existencia de extraterrestres, conexiones con fuerzas paranormales, difusión de ideas sobre campos energéticos, encarnaciones de otras vidas pasadas, programaciones mentales propias y ajenas, introducción a lecturas extrañas (Tao Te King, Tao del amor y del sexo, I Ching, métodos de control mental). Incursión por obscuros caminos espirituales. El misticismo, el ostracismo.

\section{Prácticas corporales instrumentales}

Las prácticas corporales pueden ser contempladas desde ópticas de apreciación diversas. Si las claves de lectura están localizadas en la ergonomía, la comunicación motriz, los estereotipos motrices, las técnicas del movimiento, predomina un enfoque ligado al conductismo social. Es decir, el análisis de un encadenamiento motor, el desmenuzar la complejidad de un gesto en unidades pequeñas para su estudio tiene resultados desprovistos de aspectos socioculturales. La descripción detallada de técnicas, acciones motoras, deslizamientos, son propiedades referidas a la eficacia y economía de los desplazamientos. Las capacidades de contracción y relajación muscular, la sudoración, la pilosidad del cuerpo, el análisis biomecánico de un salto con 
garrocha, atiende al cuerpo como sistema de palancas, fuerzas, poleas, resistencias. Es decir, plantea o se vale de insumos procedentes de la física para explicar esas fuerzas de acción y reacción del cuerpo y su entorno circundante, las caídas, fuerzas centrifugas y centrípetas que accionan en la marcha.

Las prácticas corporales en el ámbito disciplinar de la Educación Física están ligadas a conceptos como disponibilidad motriz, inteligencia motora, actos motores, mecanismos del acto motor, fases del aprendizaje motor, transferencia, habilidades motrices generales y básicas, capacidades condicionales coordinativas. Tareas, actividades, driles, ejercitaciones, mecánica de piernas. Hay un lenguaje codificado e hiperespecializado que otorga consistencia y espesor a un tipo de prácticas. Son prácticas corporales leídas desde la óptica de apreciación y categorización de la Educación Física. Es un discurso que irradia esas prácticas.

Lo que esta en juego en los encuentros entre practicantes del cuerpo en la ciudad es un sentido social de algún tipo. En el boxeo femenino se puede constatar con el enfoque biográfico como se construyen particulares sentidos de vida desafiando las interpelaciones de la cultura ${ }^{1}$. El skate en las plazas no es una mera ejecución repetitiva de ensayos y errores mecánicos. Algún sentido aglutina a estos practicantes y los moviliza para ocupar un territorio en la ciudad. No es solo el placer por el logro de un movimiento, es algo más, ligado a una búsqueda del sujeto de un posible sitio en la ciudad, canalizado a través del cuerpo. Es la iniciativa para no quedar al margen.

Los malabares en las calles contienen un sentido que lejos está de ser una rápida expresión corporal de juglares urbanos. No es arte callejero. Es el desesperado cuentapropismo de sujetos que encuentran una manera de generar humildes recaudaciones. En la forzada cara de alegría que nos ofrece el precario acróbata en las esquinas, se manifiesta la realidad cruda de personas que viven al día al ritmo de las fases de los semáforos. El sentido de pobreza y el montaje de estrategias de supervivencia poniendo el cuerpo en la ciudad, para que esta sea generosa y tenga piedad ante tanta miseria.

\section{Poner el cuerpo en la ciudad}

Poner el cuerpo en la ciudad, vivir la ciudad, encontrar lugares de comodidad, refugios, tugurios clandestinos, sitios de distinción, de identidad, pertenencia, configurar un clan, pertenecer a una manada. Ser ciudadano, construir la ciudadanía, se hace movilizando prácticas corporales ${ }^{2}$. El cuerpo de la frivolidad y la vanidad de los desfiles con ropas de marca en bares y cafés de las señoras pacatas, la frecuente asistencia a complejos deportivos de moda para alimentar el egocentrismo y el narcisismo radical, o protagonizar actividades corporales novedosas se explica con subjetividades peculiares.

1 Véase el análisis de la entrevista realizada con la boxeadora Vanesa Ignomiriello, donde se explicitan los pleitos existenciales que deben resolver en la construcción de una relación con una práctica corporal ligada a la lucha (CACHORRO, 2007).

2 La relevancia social que posee la "construcción de ciudadanía" para las futuras generaciones impulsó la construcción de diseños curriculares en la provincia de Buenos Aires, atendiendo a distintos ámbitos de realización prioritarios (comunicación y tecnologías, arte, estado y política, medio ambiente, recreación y deporte, salud y alimentación, sexualidad y trabajo). Es una decisión importante porque con ella se intenta cuidar a los jóvenes, acompañarlos hacia el futuro haciendo uso de derechos y obligaciones. 
Rebasar las interpretaciones de las prácticas corporales en la perspectiva exclusiva de la Educación Física y sus comportamientos motores es correrse hacia el análisis de los discursos institucionales que atraviesan y le dan consistencia y espesor ${ }^{3}$. Se hacen cuerpo, se incorporan de forma contradictoria y caótica. Son prácticas estructuradas desde matrices culturales que dirigen esas performances y la figuran una y otra vez.

El pasaje del mapa a la cartografía nos coloca en los recorridos, las mudanzas, los desmantelamientos, las refundaciones de los espacios y los tiempos de la cultura urbana. Exceden las coordenadas del croquis estático, es más que la representación gráfica del dibujo. La construcción de moradas pasajeras, las relaciones inestables con los lugares y personas, la disolución de los encuentros fortuitos y pasajeros entre desconocidos reunidos en un lapso de tiempo efímero con el pretexto de hacer deporte, jugar, entrenarse en clubes, asociaciones, plazas, alojan un espectro amplio de expectativas sociales.

Existe un contexto de realización de la cultura corporal en la ciudad, se conjugan tiempos históricos, políticos, económicos y su composición abierta establece una rica relación dialéctica con los actores. En esa relación del sujeto con la cultura puede pensarse y promover la opinión crítica sobre la aparición y desaparición de los cuerpos, la expropiación de la experiencia barrial, el secuestro del espacio público, la privatización de las instancias de recreación, el armado de fiestas infantiles seriadas por la lógica del mercado, la intromisión en la vida intima de las agencias. Es decir, analizar los procesos del cuerpo en la ciudad implica aterrizar en el corazón de las prácticas sociales y ver de que manera los ciudadanos asumen posiciones en la trama discursiva que intenta maniatarlos.

Volvamos a la pregunta ¿Dónde podemos poner el cuerpo en esta ciudad? Es importante pensar en lugares donde el cuerpo pueda manifestarse o descargarse. La plenitud, la libertad, la expansión del cuerpo y la obtención de la felicidad arrastran a los moradores a buscar sitios de realización. La fiesta, el desahogo, la risa, la celebración, los abrazos, los besos, las caricias, los mimos abonan el cuerpo más allá de los alimentos orgánicos, le ponen el alma al cuerpo, el aliento de la vida. Los sujetos acuerdan, en sus prácticas corporales, esas búsquedas de instantes efímeros de festejos colectivos. Un aplauso emocionante de espectadores en conjunto por una obra de teatro, un rezo mancomunado de devotos eclesiásticos, un grito al unísono de la parcialidad albiazul ante un gol, un cuerpo de manifestantes haciendo un reclamo ruidoso en las calles. Son postales corporales que hacen patente esa idea.

La exploración de los sujetos se multiplica ante el atravesamiento de la cultura mediante el avance de los procesos de globalización y mundialización de los patrimonios corporales. Con esas influencias se producen "nuevas sensibilidades" o una nueva “mundanidad técnica” (MARTÍN BARBERO, 2004, p. 262). En la vida ciudadana la influencia de las nuevas tecnologías, la presencia de la técnica, construye un "nuevo sensorium" con el aporte de nuevos objetos mediadores de las relaciones entre actores. Juegos electrónicos, mp3, laptops, teléfonos celulares componen otro paisaje urbano y configuran otras técnicas corporales en la ciudad.

3 En esta posible ruta de investigación se halla "mapas de la Educación Física" e "ideas para pensar la educación física", cuya agenda de temas propone un listado de prioridades no atendidas en la matriz de formación profesional del área (CACHORRO, 2009). 


\section{Interpretaciones del hacer corporal en la ciudad}

La riqueza de la ciudad invita a romper las rutas cotidianas, alterar los circuitos rutinarios y entrar a recintos no visitados. Presenciar la noche en la zona roja, entrar a bourdeles, ver el show del cabaret, recorrer las calles ardientes de travestis, transformistas, prostitutas y transexuales, ejercitar un vouyeurismo académico sin ser atrapado y capturado por los discursos de la sexualidad clandestina, puede ser una experiencia antropológica que supere los pruritos de la alta cultura del "homo academicus", en un sitio donde el cuerpo vibra, exclama, vomita, se desnuda. Es el cuerpo que conmueve, perturba y nos emociona. Siguiendo a Bernard Arcand (1993, p. 2) "el que no entro a un bourdel, algo se ha perdido".

Estudiar las prácticas corporales en la ciudad desde nuestra perspectiva significa recuperar a un lúcido Michelle De Certeau $(1996,40)$ para analizar las estrategias, técnicas, tácticas que despliegan los ciudadanos en lógicas del desempeño por descubrir los espacios. Las prácticas corporales condensan en su puesta en escena discursos institucionales. Adquieren sentido porque cobijan una subjetividad y operan en tramas intersubjetivas montando complejos dispositivos de interrelación cultural. En el cuerpo de la ciudad tenemos un referente empírico valioso para capturar sentidos y poner en marcha pesquisas de las películas que cada sujeto protagoniza cada vez que entra a distintos casilleros de la vida social.

Las prácticas corporales operacionalizan las subjetividades con toda la extensión y ambigüedad que ello implica. Están dirigidas por matrices de pensamiento, son movidas por estructuras para actuar en el mundo, están guiadas por esquemas de percepción, pensamiento y acción. No son meras ejecuciones instrumentales. A su vez, las prácticas corporales no se asumen como meras reproducciones de ideas lineales. Las prácticas corporales tienen distintos niveles de ruptura, cambio, transformación, innovación que va de la mano con una combinatoria de elementos que en su conjunto produce un destino incierto.

\section{El devenir y las proyecciones de las prácticas}

En las prácticas corporales pueden entreverse tendencias, predominios, prevalencias en los modos de ser y posicionarse en la vida. Los moldes que tienden a la conservación, a la inercia que no innova y mantiene un orden imperturbable pueden ser derrumbados por crisis estructurales y promover ciertos cambios. La regularidad es el círculo vicioso de algunos practicantes que no se apartan de una preferencia corporal. Toda la vida dedicada a procrear hijos, vivir en la delincuencia, hacer deporte, gozar del baile o manejar autos. En este tipo de historias existe una probabilidad de destinos, algunos trágicos, otros tranquilos, y eventuales aburridos. Los sujetos van mostrando su andar y en cierto sentido poseen una "biografía anticipada"4 que no sorprende en los posibles accidentes de la travesía.

4 La trayectoria de vida de un alumno en los distintos niveles del sistema educativo, reconstruida por Fabián Sagardoy (2007) en "Ezequiel: relato de vida", confirma esta crónica de un final anticipado. 
La tranquilidad monótona a veces es sucumbida por el imprevisto, el azar y la incertidumbre. En ese sentido podemos analizar la capacidad creativa, la recreación y la valentía de asumir riesgos, la audacia por repreguntarse y probar otra cosa para adaptarse a una nueva coyuntura existencial. Aquí cobra importancia los "imaginarios como patrimonios urbanos” (GARCÍA CANCLINI, 1997, p. 88) capaces de bocetar en nuestros pensamientos qué vamos a hacer y qué nos puede pasar en nuestra ciudad.

\section{Bodily practices. The translation of meanings in the city}

\section{Summary}

This research study refers to the recording and inventory-making of bodily practices enacted by youngsters in the city of La Plata, in the province of Buenos Aires, Argentina. Material collected from field study contributes to an interpretive analysis of the social meanings which are at play by youngsters through the mapping and charting of their forms of participation. This research work aims at constructing an aesthetics for the ways of observation which can enable us to follow citizenhip-building processes by means of this precious material called the body.

Keywords: Body Practices - City - Youngsters - Body

\section{Práticas corporais. Tradução de sentidos na cidade}

\section{Resumo}

O presente trabalho se refere ao registro e inventario de práticas corporais desdobradas pelos(as) jóvens na cidade de La Plata, Província de Buenos Aires. O levantamento desses materiais no trabalho de campo é aproveitado para uma análise interpretativa dos sentidos sociais postos en jogo pelos(as) jovens com o desenho de mapas e cartografias de suas formas de participação. A proposta de trabalho aspira a construção de uma estética do olhar, capaz de acompanhar os processos de construção da cidadania por meio desse precioso material chamado corpo.

Palavras-chaves: Práticas corporais - Cidade - Jovens - Corpo

\section{Referencias}

ARCAND, B. El jaguar y el oso hormiguero. Antropología de la pornografía. Buenos Aires: Nueva Visión, 1993.

BARRIOS, J. L. El cuerpo grotesco. Desbordamiento y significación. Primeras jornadas de cuerpo y cultura de la UNLP. La Plata, 17 de mayo de 2008. p. 15-30.

BLEICHMAN, S. Subjetividad en riesgo. Buenos Aires: Topia, 2005.

BOURDIEU, P. Homo Academicus. Buenos Aires: Siglo XXI, 1984.

CACHORRO, G. Mapas de la Educación Física. Revista Movimento, Porto Alegre. v. 15, n. 1, p. 261-273, feb./mar. 2009.

Sujetos juveniles deportivos y enfoque biográfico. En: AGUERO, R.; ARRUETA, C.; BURGOS, R. Sobresentidos. Estudios sobre comunicación, cultura y sociedad. San Salvador de Jujuy: EdiUnju, 2007. p. 315-340.

Proyecto de investigación El campo de las prácticas corporales en la ciudad de La Plata. FHCE. UNLP. Programa de Incentivos a la Investigación. Disponible en: 
http://www.fahce.unlp.edu.ar/investigacion/proyectos-deinvestigacion/elcampodelaspracticascorporalesenlaciudaddelaplata. Acceso en: 7 de junio de 2009.

DE CERTEAU, M. La invención de lo cotidiano. Artes de hacer. Distrito Federal: UIA, 1996.

DUSCHATSKY, S. Notas sobre la relación entre escuela y subjetividades juveniles. Revista Anales de la Educación Común, DGCEPBA, Buenos Aires, Año 1, n. 1-2, p. 213-227, sep. 2005.

GAGLIANO, R. Esferas de la experiencia adolescente Revista Anales de la Educación Común, DGCEPBA, Buenos Aires, año 1, n. 1-2, p. 204-212, sep. 2005.

GALLISOT, R. Bajo la identidad el proceso de identificación. Traducción de Alberto Giménez. Seminario de relatos de vida. Maestría en Desarrollo Educativo UPN, Distrito Federal, 1987.

GARCÍA CANCLINI, N. Imaginarios Urbanos. Buenos Aires: Eudeba, 1997.

LACARRIEU, M.; PALLINI, V. Buenos Aires imaginada. Bogotá: Convenio Andres Bello, 2007.

LEVINAS, E. Totalidad e infinito. Ensayo sobre exterioridad. Salamanca: Sigueme, 1987.

MAFFESOLI, M. El nomadismo. Vagabundeos iniciáticos. Buenos Aires: FCE, 2004.

MARTÍN BARBERO, J. El oficio de cartógrafo. Travesías latinoamericanas de la comunicación en la cultura. Buenos Aires: FCE, 2004.

SAGARDOY, F. Ezequiel: relato de vida. Revista de EF \& $C$, FHCE, UNLP La Plata, año 9, n. 7, p. 205-214, dic. 2007.

SILVA, A. Polvos de ciudad. Bogotá: La balsa, 2005.

VIRILIO, P. La velocidad de la liberación. Buenos Aires: Manantial, 1997.

Recebido em: 26 de maio de 2009

Revisado em: 05 de junho de 2009

Aprovado em: 23 de junho de 2009

\section{Endereço para correspondência}

gcachorro@yahoo.com.

Gabriel Armando Cachorro

Facultad de Humanidades y Ciencias de la Educación. 48 e/ 7 y 8 s/n.

La Plata, Bs. As. Argentina 